\title{
Uniportal video-assisted thoracoscopic surgery lobectomy with systematic nodal dissection for the treatment of stage IIIA (N2) non-small cell lung cancer: a case report
}

\author{
Youngkyu Moon, Si Young Choi \\ Department of Thoracic \& Cardiovascular Surgery, Eunpyeong St. Mary's Hospital, College of Medicine, The Catholic University of Korea, Seoul, \\ Republic of Korea \\ Correspondence to: Youngkyu Moon, MD, PhD. Department of Thoracic \& Cardiovascular Surgery. Eunpyeong St. Mary's Hospital, College of \\ Medicine, The Catholic University of Korea, 1021, Tongil-ro, Eunpyeong-gu, Seoul 03312, Republic of Korea. Email: mykae@catholic.ac.kr.
}

\begin{abstract}
Uniportal video-assisted thoracoscopic surgery (VATS) has been considered more difficult than multiportal VATS because the operation is performed by inserting 2 or 3 surgical instruments and the thoracoscope into only 1 incision. Therefore, it has been thought that uniportal VATS is not suitable for the treatment of stage IIIA (N2) non-small cell lung cancer (NSCLC). However, the uniportal technique has evolved considerably. Standardized surgical methods make it easy to perform, and the development of surgical instruments and equipment allows for more sophisticated operations. In our institution, uniportal VATS is applied regardless of stage by the author, and complete resection has been successfully performed in N2 disease. One of them is introduced here. A 65-year-old female patient had left upper lobe (LUL) lung adenocarcinoma and left paratracheal, subaortic, and interlobar lymph node metastases. Successful complete resection was achieved with uniportal VATS lobectomy and systematic nodal dissection. The anesthesia time was 225 minutes, and the operative time was 162 minutes. There were no postoperative complications. The chest tube was removed on day 3 postoperatively and the patient was discharged on day 8 . The pathologic stage was T2aN2M0. The number of dissected lymph nodes was 52. After surgery, the patient received sequential adjuvant chemotherapy and radiotherapy. The patient is now well over 10 months without any recurrences. In our experience, uniportal VATS lobectomy with systematic nodal dissection in operable N2 lung cancer is a sufficiently applicable operation.
\end{abstract}

Keywords: Lung cancer; stage IIIA; uniportal video-assisted thoracoscopic surgery (uniportal VATS); case report

Received: 12 March 2020; Accepted: 23 April 2020; Published: 25 February 2021.

doi: $10.21037 /$ ccts-20-56

View this article at: http://dx.doi.org/10.21037/ccts-20-56

\section{Introduction}

Early stage non-small cell lung cancer (NSCLC) is usually treated with minimally invasive surgery. Video-assisted thoracoscopic surgery (VATS) is the most commonly used minimally invasive surgery. Most hospitals perform multiportal (3-4 ports) VATS. However, the development of new instruments and surgical techniques has reduced the number of ports, resulting in 2-port VATS and eventually uniportal VATS, which is considered a form of evolution of multiportal VATS (1). Some studies reported that uniportal VATS has the advantages of less postoperative pain, faster recovery, decreased length of hospital stay, and potential earlier administration of adjuvant therapy than multiportal VATS $(2,3)$. The advantages result from the use of only 1 intercostal space and more anterior placement of the incision than in multiportal VATS.

Despite the many advantages of uniportal VATS, most surgeons who conduct uniportal VATS prefer to apply it primarily to early stage NSCLC, because they think that uniportal VATS is a difficult surgical technique to master. Especially in the presence of lymph node metastasis, there is a perception that it is difficult to access the deep 
A Timeline and duration of each treatment

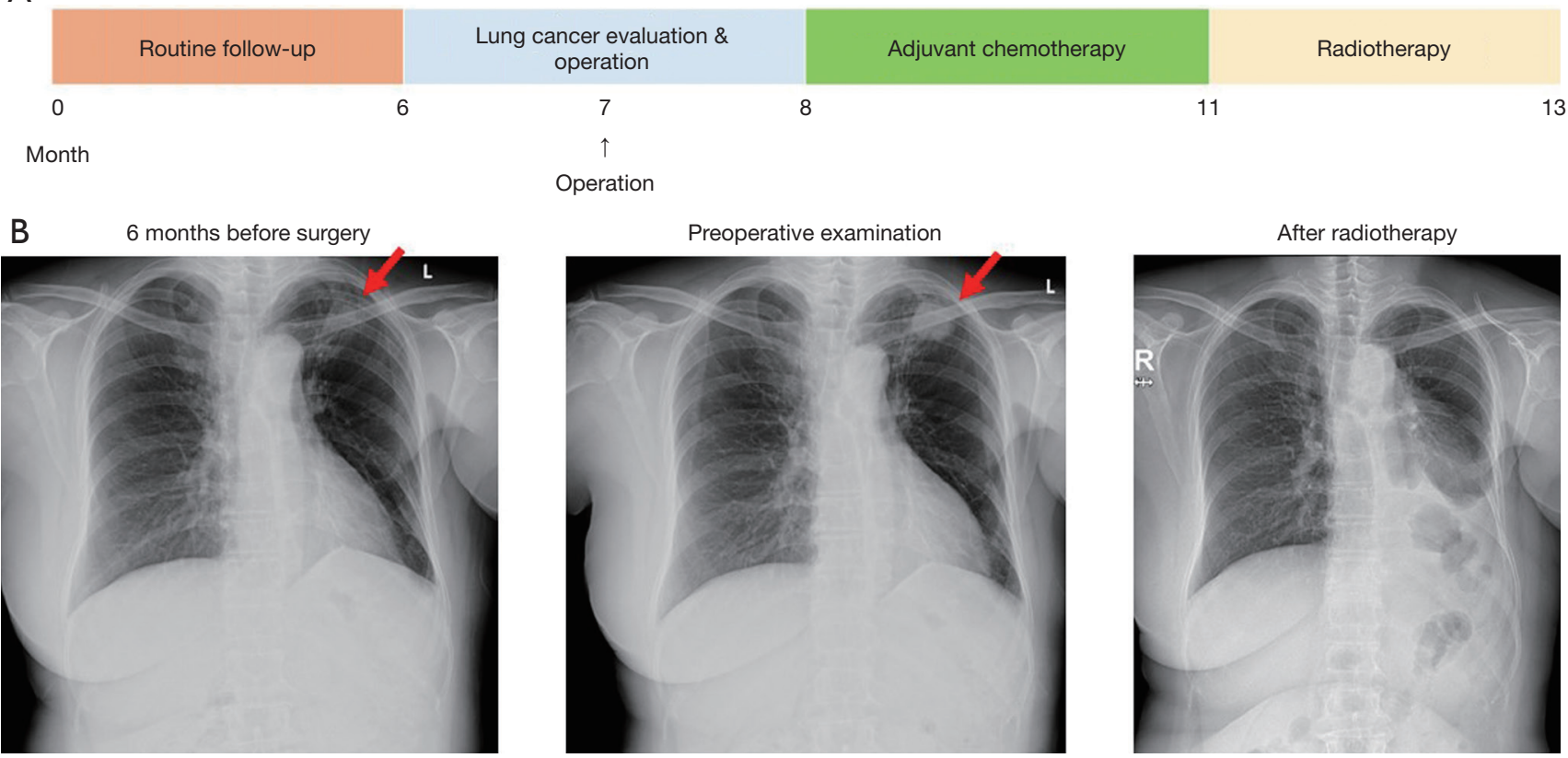

Figure 1 (A) Timeline and duration of each treatment and (B) chest plain radiograph before and after treatment.

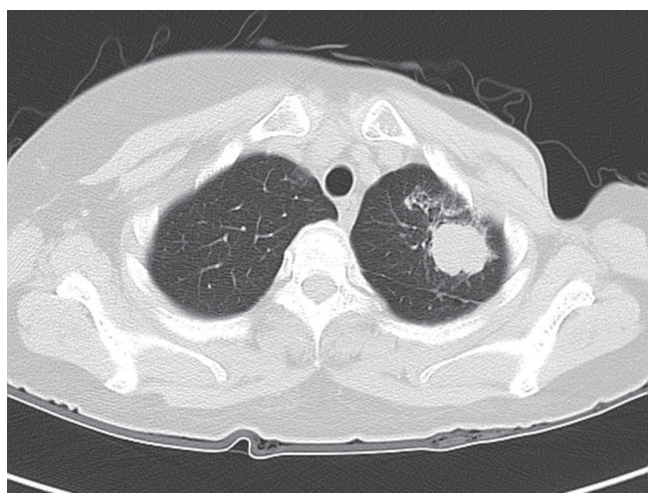

Figure 2 Chest computed tomography image shows an approximately $3-\mathrm{cm}$ solid lung mass in the left upper lobe apical segment.

and narrow mediastinal nodes using uniportal VATS because of the limited field of view, angle of operation, and the sophisticated lymph node dissection required. Therefore, multiportal VATS or open thoracotomy is preferred for surgery in stage II or III NSCLC. However, with the growing experience and skills of uniportal VATS lobectomy, uniportal VATS is now being applied to advanced NSCLC (4). So far, uniportal VATS for the treatment of stage IIIA (N2) NSCLC is a challenging procedure. We report a successful case of stage IIIA (N2)
NSCLC treated with uniportal VATS. We present the case in accordance with the CARE reporting checklist (available at https://ccts.amegroups.com/article/view/10.21037/ccts$20-56 / \mathrm{rc})$.

\section{Case presentation}

A 65 -year-old female patient visited our hospital with a suspicion of lung cancer in the left upper lobe (LUL) of the lung. Ten years ago, she underwent mastectomy of left breast and postoperative radiotherapy for breast cancer. After treatment for breast cancer, the patient had regular checkups including a plain chest radiograph every 6 months without any evidence of recurrence. She had no symptoms or signs of disease. Six months ago, a plain radiograph of the chest showed radiation pneumonitis in the LUL, which was not unlike previous findings. However, there was a newly developed lung mass in the most recent chest $\mathrm{X}$-ray where the radiation pneumonitis was present (Figure 1). Chest computed tomography (CT) with contrast demonstrated an approximately $3-\mathrm{cm}$ solid lung mass on the LUL apical segment (Figure 2). She underwent CT-guided percutaneous core needle biopsy and the mass was diagnosed as lung adenocarcinoma. In the F-18 fluorodeoxyglucose (FDG) positron emission tomography (PET) scan, the maximum standardized uptake value (SUVmax) of the 

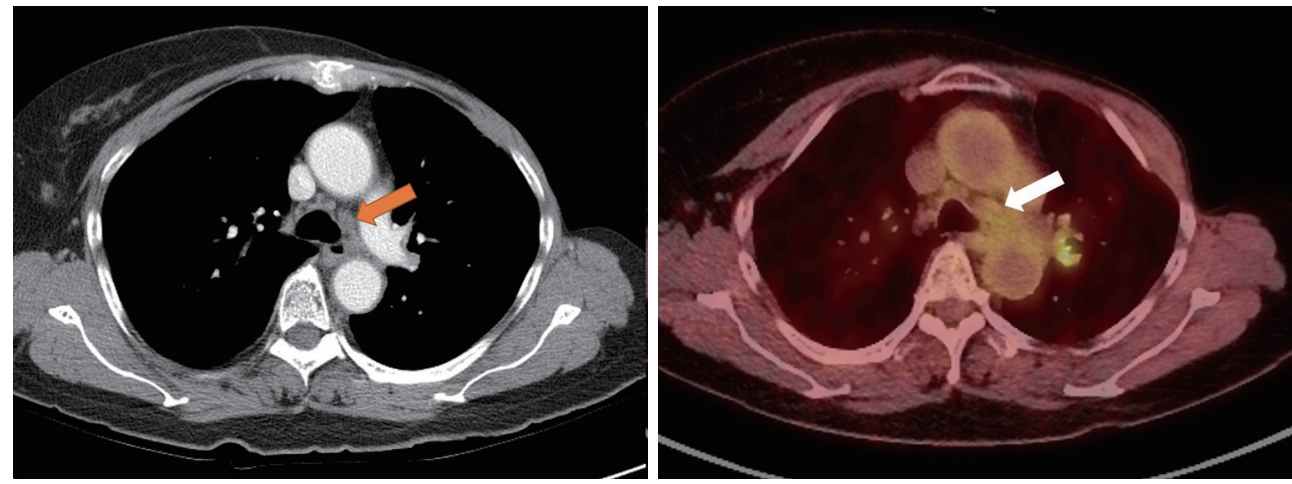

Figure 3 Enlarged left paratracheal lymph node on chest computed tomography and positron emission tomography scan.

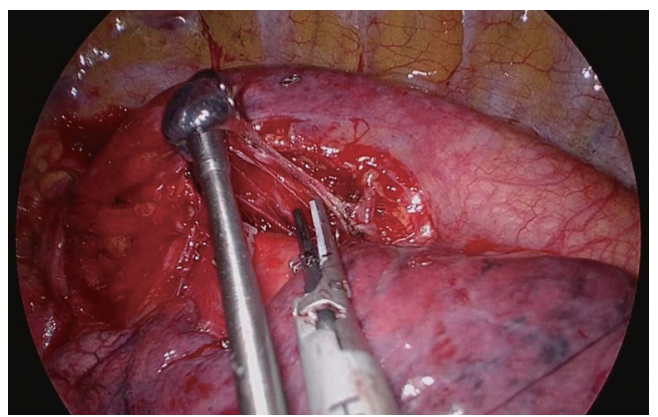

Figure 4 Left subaortic lymph node dissection using suction device for pushing or pulling without grasping the lymph node.

tumor was 15.6. The left paratracheal lymph node (\#4L) was enlarged with mild FDG uptake (Figure 3), but the endobronchial ultrasonography (EBUS) and transbronchial needle aspiration (TBNA) biopsy results were negative for malignancy. The left subaortic lymph node (\#5) and interlobar lymph node (\#11) were also enlarged and the interlobar lymph node had intense FDG uptake on PET scan. The clinical stage was T2aN1orN2M0 and curative surgery was chosen with the patient's consent.

The patient underwent uniportal VATS LUL lobectomy with systematic nodal dissection including left paratracheal lymph node (\#4L) dissection. The patient position and anesthesia were the same as that of conventional VATS. A skin incision was made in the fifth intercostal space between the midaxillary and anterior axillary lines. The incision length was $4 \mathrm{~cm}$. The 30 -degree highdefinition thoracoscope was located at the top of the working wound. An energy device (HARMONIC ACE,
J\&J Pharmaceuticals) and curved long suction device (SCANLAN Wolf Suction Instrument 9009-912, Scanlan International) were located in the middle of the wound. The lung was retracted with a lung-grasping clamp that was located in the lowest portion of the working wound. Using the curved suction device and the straight energy device, the surgeon achieved an unobscured field of view. The suction device both aspirated the surrounding blood and pushed or pulled the surrounding tissues, facilitating tissue dissection. Large vessels were divided with staplers. Small vessels were ligated with silk string or clips and divided with the energy device. The operation began with a hilar dissection, and the superior pulmonary vein was exposed. Then, by changing the angle of the stapler to orient the anvil into the proximal portion of the vessel, the vessels were divided. After dividing the superior pulmonary vein, the anterior and apical branches of the pulmonary artery was divided by a stapler at the same angle. After fissure division, the lingular segmental artery and posterior segmental artery were divided by a stapler and surgical clips. Lymph node dissection around the lobar bronchus was conducted, and the bronchus was divided by a stapler. Systematic nodal dissection was performed by en bloc resection of the lymph nodes and adjacent fat tissues. The lymph nodes were dissected using a nongrasping technique that involved dissection of the surrounding tissue with an energy device and use of a suction device for pushing or pulling without grasping the lymph nodes. Left paratracheal lymph nodes (\#4L) were enlarged and they were exposed after subaortic lymph node dissection (\#5) (Figure 4). The paratracheal node was also removed by en bloc resection (Figure 5). In order to sufficiently expose the subcarinal node, the bronchus and aorta were retracted using 2 curved suction devices and then en bloc removal was performed by using an energy 

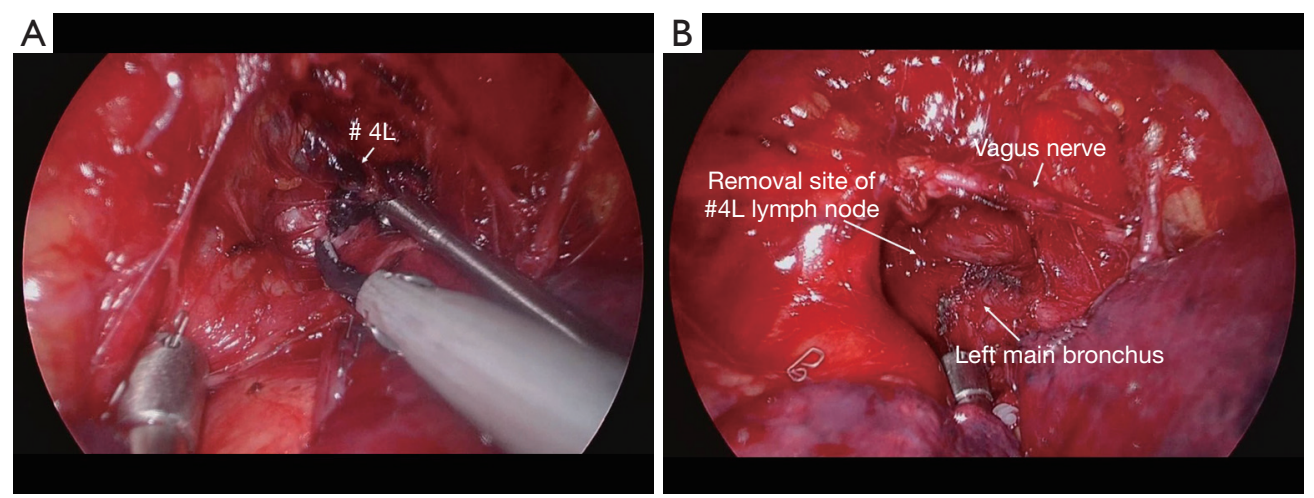

Figure 5 (A) Before and (B) after removal of the left paratracheal lymph node.
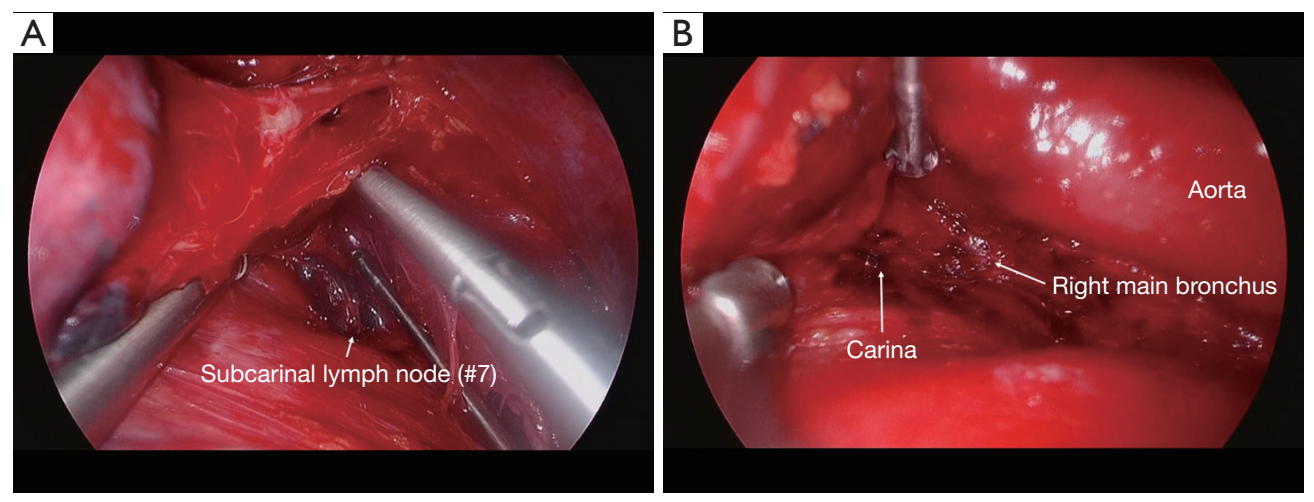

Figure 6 (A) Before and (B) after removal of the subcarinal lymph node. After nodal dissection, the right main bronchus was exposed.

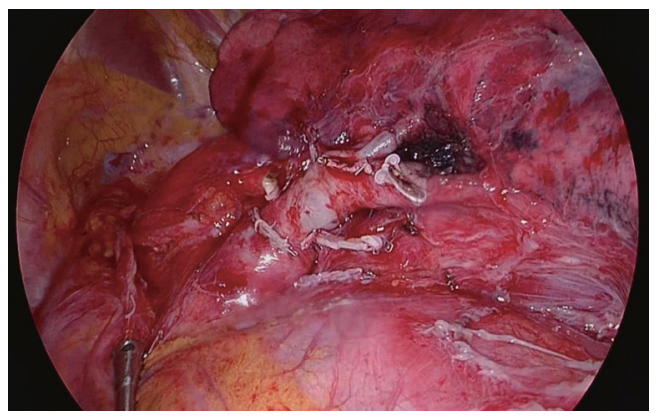

Figure 7 Left lower lobe and remnant stumps after left upper lobectomy.

device until the right main bronchus was exposed (Figure 6). After left upper lobectomy and systematic nodal dissection (Figure 7), a chest tube was inserted into the thoracic cavity through the working incision, and the incision site was closed layer by layer. No complications occurred during the operation. The anesthesia time was 225 minutes, and the operative time was 162 minutes. There were no postoperative complications. The chest tube was removed on day 3 postoperatively and the patient was discharged on day 8 . The final diagnosis after analysis of the pathologic specimen was lung adenocarcinoma stage T2aN2M0. The number of dissected lymph nodes was $52(\mathrm{~N} 1,11 ; \mathrm{N} 2,41)$. Left paratracheal and subaortic lymph nodes had metastatic cancer cells. Although the cancer was completely resected, the patient underwent sequential adjuvant chemotherapy and radiotherapy (Figure 1). One month after surgery, the patient received paclitaxel $(250 \mathrm{mg})$ intravenously followed by infusion of carboplatin $(600 \mathrm{mg})$, with each administered on day 1 every 3 weeks for four cycles. Then, the patient received radiation therapy for 5 weeks (50 Gray/5 fractions) after chemotherapy. Ten months have passed since the surgery, and the patient is doing well with regular surveillance without cancer recurrence.

All procedures performed in this study were in accordance with the ethical standards of the institutional 
and national research committees and with the Helsinki Declaration (as revised in 2013). Written informed consent was obtained from the patient for publication of this case report and any accompanying images. A copy of the written consent is available for review by the editorial office of this journal.

\section{Discussion}

According to the NCCN (National Comprehensive Cancer Network) guideline for NSCLC (version 2.2020), VATS should be strongly considered for patients with no anatomic or surgical contraindications, as long as there is no compromise of the standard oncologic and dissection principles of thoracic surgery. In lung cancer, metastasis mainly occurs in the lymph nodes, so systematic nodal dissection is very important during surgery (5). Several studies have reported good oncologic outcomes only if sufficient lymph node dissection has been achieved (6-8). Therefore, in patients with suspected lymph node metastasis, some surgeons choose open thoracotomy for sophisticated lymph node dissection to improve oncologic outcomes, although VATS has a better surgical outcome (9). Nevertheless, experienced VATS surgeons perform VATS lobectomy with techniques similar to open thoracotomy for advanced lung cancer. With more experience and continued development of better surgical techniques and instruments, the constraints of minimally invasive surgery are gradually decreasing (10). The uniportal technique also has evolved considerably $(3,11,12)$. The 30 -degree thoracoscope placed at the top of the wound provides an optimal field of view similar to that of actual open thoracotomy. Moreover, if the surgery is done in the order determined for each lobe using a long curved suction device and energy device, the operation can be performed relatively easily and quickly. Also, if the suction device is used for aspiration as well as for multiple other functions (e.g., to hold, dissect, expose, and compress), there is no need for an additional port. During lymph node dissection, the curved suction device can be used to push or pull the lymph node to dissect the tissue around the lymph node with an energy device for en bloc resection. The node was less likely to break.

The author performed over 100 cases of uniportal VATS lobectomy for the treatment of lung cancer from 2018-2019. In 2017, he attended a uniportal VATS training program at Tongji University Shanghai Pulmonary Hospital in Shanghai, China (13). The hospital is an ultra-highvolume center for thoracic surgery where more than 10,000 lung cancer resections are performed annually. There, the author learned the techniques of uniportal VATS lobectomy and segmentectomy by observing the operations of Dr. Diego Gonzalez-Rivas. In Shanghai, he found that uniportal VATS lobectomy was never a more difficult operation than multiportal VATS or open thoracotomy. The author performed uniportal VATS in all patients with operable lung cancer regardless of stage, and has never converted to multiportal VATS or open thoracotomy.

This case had several limitations. First, only 1 case has been presented in this report. Second, this case does not represent all stage IIIA (N2) NSCLC. In fact, N2 disease exists in various patterns. Therefore, there may be N2 disease which uniportal VATS is impossible. More cases will be collected and we will present the surgical and oncologic outcomes of uniportal VATS in patients with stage IIIA (N2) NSCLC.

\section{Conclusions}

In our experience, uniportal VATS lobectomy with systematic nodal dissection in operable Stage IIIA (N2) lung cancer was a sufficiently applicable operation. Systematic nodal dissection for lung cancer surgery can also be performed properly with uniportal VATS. It is hoped that the future will bring wider application of uniportal VATS along with better results for the treatment of lung cancer.

\section{Acknowledgments}

Funding: None.

\section{Footnote}

Reporting Checklist: The authors have completed the CARE reporting checklist. Available at https://ccts.amegroups. com/article/view/10.21037/ccts-20-56/rc

Conflicts of Interest: Both authors have completed the ICMJE uniform disclosure form (available at https://ccts.amegroups. com/article/view/10.21037/ccts-20-56/coif). YM serves as an unpaid editorial board member of Current Challenges in Thoracic Surgery from January 2019 to December 2021. The other author has no conflicts of interest to declare.

Ethical Statement: The authors are accountable for all aspects of the work in ensuring that questions related to the accuracy or integrity of any part of the work are 
appropriately investigated and resolved. All procedures performed in this study were in accordance with the ethical standards of the institutional and national research committees and with the Helsinki Declaration (as revised in 2013). Written informed consent was obtained from the patient for publication of this case report and any accompanying images. A copy of the written consent is available for review by the editorial office of this journal.

Open Access Statement: This is an Open Access article distributed in accordance with the Creative Commons Attribution-NonCommercial-NoDerivs 4.0 International License (CC BY-NC-ND 4.0), which permits the noncommercial replication and distribution of the article with the strict proviso that no changes or edits are made and the original work is properly cited (including links to both the formal publication through the relevant DOI and the license). See: https://creativecommons.org/licenses/by-nc-nd/4.0/.

\section{References}

1. Sihoe AD. The evolution of minimally invasive thoracic surgery: implications for the practice of uniportal thoracoscopic surgery. J Thorac Dis 2014;6:S604-17.

2. Hirai K, Takeuchi S, Usuda J. Single-incision thoracoscopic surgery and conventional video-assisted thoracoscopic surgery: a retrospective comparative study of perioperative clinical outcomesdagger. Eur J Cardiothorac Surg 2016;49 Suppl 1:i37-41.

3. Gonzalez-Rivas D, Sihoe ADL. Important Technical Details During Uniportal Video-Assisted Thoracoscopic Major Resections. Thorac Surg Clin 2017;27:357-72.

4. Bulgarelli Maqueda L, Garcia-Perez A, Minasyan A, et

doi: $10.21037 /$ ccts-20-56

Cite this article as: Moon Y, Choi SY. Uniportal videoassisted thoracoscopic surgery lobectomy with systematic nodal dissection for the treatment of stage IIIA (N2) non-small cell lung cancer: a case report. Curr Chall Thorac Surg 2021;3:11. al. Uniportal VATS for non-small cell lung cancer. Gen Thorac Cardiovasc Surg 2020;68:707-15.

5. Watanabe S, Asamura H. Lymph node dissection for lung cancer: significance, strategy, and technique. J Thorac Oncol 2009;4:652-7.

6. Dai J, Liu M, Yang Y, et al. Optimal Lymph Node Examination and Adjuvant Chemotherapy for Stage I Lung Cancer. J Thorac Oncol 2019;14:1277-85.

7. Krantz SB, Lutfi W, Kuchta K, et al. Improved Lymph Node Staging in Early-Stage Lung Cancer in the National Cancer Database. Ann Thorac Surg 2017;104:1805-14.

8. Park JK, Moon Y. Prognosis of upstaged N1 and N2 disease after curative resection in patients with clinical N0 non-small cell lung cancer. J Thorac Dis 2019;11:1202-12.

9. Whitson BA, Groth SS, Duval SJ, et al. Surgery for earlystage non-small cell lung cancer: a systematic review of the video-assisted thoracoscopic surgery versus thoracotomy approaches to lobectomy. Ann Thorac Surg 2008;86:200816; discussion 2016-8.

10. Curcio C, Amore D, Casazza D, et al. Thoracoscopic lobectomy for lung cancer in challenging cases: technical aspects. Curr Chall Thorac Surg 2019;1:25.

11. Hernandez-Arenas LA, Purmessur RD, Gonzalez-Rivas D. Uniportal video-assisted thoracoscopic segmentectomy. J Thorac Dis 2018;10:S1205-14.

12. Gonzalez-Rivas D, Fieira E, Delgado M, et al. Uniportal video-assisted thoracoscopic lobectomy. J Thorac Dis 2013;5 Suppl 3:S234-45.

13. Sihoe ADL, Gonzalez-Rivas D, Yang TY, et al. Highvolume intensive training course: a new paradigm for video-assisted thoracoscopic surgery education. Interact Cardiovasc Thorac Surg 2018;27:365-71. 